\title{
Avaliando a Geração de Buzz de Issues em Comunidades de Softwares de Código-Aberto
}

\author{
Igor Muzetti Pereira \\ Universidade Federal de Ouro Preto - UFOP \\ João Monlevade, Brasil \\ igormuzetti@ufop.edu.br
}

\begin{abstract}
Resumo - O desenvolvimento de sistemas open-source constrói implicitamente uma comunidade que contribui, reporta problemas e compartilha dúvidas entre os seus colaboradores. Dessa forma, diversas plataformas auxiliam essas comunidades, tais como o Github e o StackOverflow, responsáveis por hospedar código-fonte, gerir problemas relacionadas a bugs, fazer controle de versão e solucionar dúvidas de usuários. Entretanto, pouco se sabe sobre a relação que existe entre ambas as comunidades, especificamente o buzz gerado entre elas a partir de problemas encontrados no códigofonte. Dessa forma, neste trabalho estuda-se o buzz gerado no StackOverflow a partir de issues reportadas no Github, bem como o impacto dessas perguntas dentro da comunidade do StackOverflow. Para tanto, foram analisados oito sistemas JavaScript, divididos em dois grupos: populares e não populares, e suas issues reportadas em um mês. Como resultado, observou-se a pequena interação entre ambas as comunidades, assim como um baixo impacto dos temas discutidos no StackOverflow relacionados aos problemas reportados no Github.
\end{abstract} issues.

Palavras-chave - stackoverflow; github; open-source; buzz;

\section{INTRODUÇÃO}

A grande quantidade de informações às quais os colaboradores são expostos por meio das redes sociais está mudando a maneira como eles colaboram, se comunicam e aprendem. Isso impacta em última análise, a maneira como eles desenvolvem sistemas computacionais [Storey et al, 2010]. A participação de usuários no StackOverflow quanto no Github podem ser consideradas como atividades em redes sociais. Enquanto o StackOverflow avalia a contribuição do participante em termos de pontos de reputação e medalhas, a plataforma encoraja os participantes a fazerem perguntas inteligentes e darem respostas que satisfaçam os usuários que as fizeram [Capilippi et al, 2013] [Dabbish et al, 2012]. Assim, os usuários do Github estão cientes de serem observados por outros desenvolvedores, e essa consciência influencia como eles se comportam e gerenciam suas contas, inclusive para melhorarem seus currículos [Montadanon et al, 2020].

Existe uma diversidade de plataformas de redes sociais, que abordam um conjunto de atividades dos usuários. Comumente, um único usuário tem várias contas em diferentes redes sociais. Por isso, se torna cada vez mais importante vincular contas distribuídas pertencentes ao mesmo usuário de várias fontes, o que pode beneficiar várias aplicações. A agregação de perfis de usuário pode melhorar de alguma maneira sistemas de recomendação, como demonstra o estudo de Abel et al (2013). Badashian et al (2014), consideraram a influência que cada usuário possui entre duas plataformas. Eles exploraram os recursos fornecidos pelo StackOverflow (votos e questões) e Github (commits, projetos e issues).

O Github é uma plataforma de hospedagem de códigofonte com controle de versão usando o Git. Ele é bastante utilizado por desenvolvedores de software para divulgação de seus trabalhos ou para que outros desenvolvedores contribuam com o projeto. Ele também promove uma fácil comunicação através de recursos que relatam problemas ou mesclam repositórios remotos. O Stackoverflow ${ }^{1}$ é um website que apresenta perguntas e respostas em uma grande quantidade de tópicos de desenvolvimento de software. Este website serve como plataforma para que os usuários façam perguntas e também as respondam. Mas além disso podem, através do registro e da participação ativa, votar em questões e respostas mais ou menos úteis de modo similar a um wiki.

Ambas plataformas são comumente utilizadas por comunidades de desenvolvedores de software de código aberto. Treude et al (2011), apresentaram um estudo que discute como os desenvolvedores fazem perguntas e respondem no StackOverflow. Robbinson et al (2016), mostraram alguns comportamentos de desenvolvedores a partir da mineração de repositórios de código aberto, como o Github.

Sendo assim, este trabalho tem como objetivo geral avaliar através de um estudo empírico a geração de buzz de issues do Github em questões do StackOverflow. Foram selecionados oito repositórios de código aberto do Github, classificados em dois grupos. O primeiro grupo conta com quatro projetos populares e o outro com quatro projetos não tão populares. Após a análise de correlação, nenhuma questão do segundo grupo continha issues correspondentes. Foram consideradas somente issues e questões criadas no período de 15/04/18 até 15/05/18 (30 dias). O objetivo deste estudo foi dividido em quatro questões de pesquisa:

- QPA. Com que frequência issues do Github são discutidas no StackOverflow?

- QPB. Qual o impacto da discussão de issues no StackOverflow?

- QPC. Existe uma relação entre as características dos sistemas e o buzz gerado no StackOverflow?

- QPD. Qual o perfil das pessoas que perguntam sobre issues no StackOverflow?

O restante deste documento está organizado da seguinte forma. Seção 2 descreve a metodologia proposta e seguida

1 https://stackoverflow.com/ 
para o desenvolvimento deste estudo. Seção 3 apresenta e discute os principais resultados obtidos. Seção 4 discute os trabalhos correlatos. Seção 5 relata as ameaças à validade identificadas e discute as principais decisões para mitigá-las. Seção 6 conclui este estudo.

\section{Metodologia}

Com o objetivo de responder às questões de pesquisa propostas, foram analisados, oito sistemas JavaScript hospedados no Github. Nesta seção, será apresentada a metodologia utilizada neste estudo: inicialmente, serão caracterizados os sistemas selecionados, bem como os critérios de escolha dos mesmos (Seção 2.1); em seguida, serão descritas as métricas utilizadas em cada questão de pesquisa estudada (Seção 2.2).

\section{A. Seleção de Sistemas}

Neste estudo foram analisadas as issues de oito repositórios de sistemas open-source encontrados no Github. Para tanto, foram selecionados repositórios populares e considerados ativos, de acordo com os seguintes critérios:

Número de estrelas. Foram selecionados sistemas populares e não-populares, com pelo menos 100 estrelas.

Número de commits. Foram filtrados sistemas com pelo menos um commit no último mês da pesquisa, representando o estado ativo do mesmo.

Dessa forma, os grupos de sistemas populares e nãopopulares é composto de quatro repositórios, respectivamente. A Tabela 1 apresenta a caracterização destes repositórios, por meio do número de estrelas, contribuidores e commits. No grupo dos sistemas populares, destaca-se o sistema FACEBOOK / REACT, com 95.893 estrelas, 1.179 contribuidores e 9.888 commits, representando também o repositório mais popular de todo o Github no período da consulta. Por outro lado, no grupo dos sistemas não-populares, destaca-se o sistema HBM/MDCOMPONENTS , com apenas 101 estrelas, 6 contribuidores e 740 commits.

\begin{tabular}{|l|c|c|c|}
\hline \multicolumn{1}{|c|}{ Nome } & \#Estrelas & $\begin{array}{c}\text { \#Contribui } \\
\text { dores }\end{array}$ & \#Commits \\
\hline $\begin{array}{l}\text { Facebook/ } \\
\text { React }\end{array}$ & 95.893 & 1.179 & 9.888 \\
\hline $\begin{array}{l}\text { Angular/ } \\
\text { Angular.JS }\end{array}$ & 58.430 & 1.596 & 8.777 \\
\hline $\begin{array}{l}\text { JQuery/ } \\
\text { JQuery }\end{array}$ & 48.946 & 271 & 6.327 \\
\hline $\begin{array}{l}\text { Meteor/ } \\
\text { Meteor }\end{array}$ & 39.789 & 387 & 20.897 \\
\hline $\begin{array}{l}\text { Greglobinsk } \\
\text { i/Gatsby- } \\
\text { Starter- } \\
\text { Personal- }\end{array}$ & 171 & 271 & 6.327 \\
\hline
\end{tabular}

\begin{tabular}{|l|c|c|c|}
\hline Blog & 128 & 6 & 186 \\
\hline $\begin{array}{l}\text { CacheContr } \\
\text { ol/Hippie- } \\
\text { Swagger }\end{array}$ & 102 & 2 & 78 \\
\hline $\begin{array}{l}\text { Marcrobled } \\
\text { o/ } \\
\begin{array}{l}\text { Savegame- } \\
\text { Editors }\end{array}\end{array}$ & 101 & 6 & 740 \\
\hline $\begin{array}{l}\text { HBM/MD- } \\
\text { Components }\end{array}$ & & 6 & \\
\hline
\end{tabular}

Tabela 1: Caracterização dos Repositórios Populares e Não-Populares Analisados

\section{B. Definição e Extração de Métricas}

A fim de analisar cada um dos repositórios estudados e mensurar valores que auxiliem nas discussões das questões de pesquisa propostas, foram definidas quatro métricas de interesse, descritas a seguir:

Total de Issues. Quantidade total de issues reportadas no Github para cada repositório analizado, durante o intervalo investigado de 30 dias (entre os dias 15/04/2018 15/05/2018). Para coleta dos valores, foram mineradas tais issues diretamente dos repositórios analisados, considerando aquelas com estado open e closed. Foram analisadas, por fim, 491 issues.

Total de Questões Relacionadas. Número de questões encontradas no StackOverflow que são relacionadas com issues reportadas no Github, para cada repositório. Essa métrica foi coletada manualmente, através da análise de cada uma das issues observadas, e a posterior busca de questões relacionadas ao tema da issue no StackOverflow. Assim, das 491 issues avaliadas, 12 (2,4\%) perguntas foram identificadas no StackOverflow.

Total de Votos. Quantidade total de votos que uma questão do StackOverflow recebe dos usuários da plataforma. O voto é uma forma de favoritar uma questão, revelando a sua relevância dentro da comunidade e destacando o quão útil aquela discussão é para os membros da comunidade. Esse valor foi coletado através de consulta manual, após a identificação das questões relacionadas às issues.

Total de Respostas. Número de respostas recebidas para cada questão identificada no StackOverflow. Esse valor também revela a relevância da pergunta proposta, bem como o buzz gerado pela pergunta dentro da comunidade do StackOverflow.

\section{RESUlTADOS}

Essa seção apresenta os principais resultados encontrados neste estudo. Seção 3.1 responde a questão de pesquisa QPA. Seção 3.2 responde a questão de pesquisa QPB. Seção 3.3 responde a questão de pesquisa QPC. Seção 3.4 responde a questão de pesquisa QPD. 


\section{A. Com que frequência issues do Github são discutidas no StackOverflow?}

Nesta questão de pesquisa investiga-se qual frequência com que issues reportadas em repositórios de projetos relacionam-se com questões levantadas no StackOverflow.

A Tabela 2 resume esses dados para as issues reportadas no intervalo previamente definido. Vale ressaltar que o percentual de questões relacionadas foi calculado baseado na razão do número de issues que possuem questões relacionadas no StackOverflow pelo total de issues encontrados no sistema. Nos casos em que uma issue possui mais de uma questão relacionada ela foi contabilizada uma única vez no momento realizar o cálculo do percentual. Desta forma, como apenas o FACEBOOK/REACT apresentou um caso em que foram encontradas três questões no StackOverflow referente a uma mesma issue, o valor de issues com questões relacionadas utilizado no cálculo do percentual do FACEBOOK/REACT foi 6.

Em três dos quatro repositórios populares verifica-se a existência de questões do StackOverflow relacionadas com issues, embora em baixo percentual. No repositório JQUERY/JQUERY, embora popular, não foram encontradas questões relacionadas à issues. Em todos os sistemas nãopopulares não foram encontradas correspondências entre questões do StackOverflow e suas respectivas issues. Em suma, verifica-se que, em um total de 491 issues reportadas nos repositórios analisados, foram identificadas apenas 12 questões relacionadas.

\begin{tabular}{|c|c|c|}
\hline Repositório & \#Issues & $\begin{array}{c}\text { \#Questões } \\
\text { relacionadas }\end{array}$ \\
\hline Facebook/React & 224 & $6(2,7 \%)$ \\
\hline $\begin{array}{c}\text { Angular/ } \\
\text { AngularJS }\end{array}$ & 90 & $2(2,2 \%)$ \\
\hline JQuery/JQuery & 42 & $0(0 \%)$ \\
\hline Meteor/Meteor & 112 & $0(1,8 \%)$ \\
\hline $\begin{array}{c}\text { Greglobinski/ } \\
\text { Gatsby-Starter- } \\
\text { Personal-Blog }\end{array}$ & 13 & $0(0 \%)$ \\
\hline $\begin{array}{c}\text { CacheControl/ } \\
\text { Hippie-Swagger }\end{array}$ & 0 & $0(0 \%)$ \\
\hline $\begin{array}{c}\text { Marcrobledo/ } \\
\text { Savegame-Editors }\end{array}$ & 9 & $0(0 \%)$ \\
\hline $\begin{array}{c}\text { HBM/MD- } \\
\text { Components }\end{array}$ & 1 & $\mathbf{1 2}(\mathbf{1 , 2 2 \% )}$ \\
\hline \begin{tabular}{c} 
Total \\
\hline
\end{tabular}
\end{tabular}

Tabela 2: Quantidade de Questões Relacionadas a Issues

A seguir são apresentados três exemplos de questões do StackOverflow relacionadas a issues do Github coletadas no intervalo de tempo definido na Seção 2.2.
Exemplo \#1. A Figura 1 mostra a issue \#12761 ${ }^{2}$ pertencente ao repositório FACEBOOK/REACT e sua respectiva questão relacionada no StackOverflow ${ }^{3}$. Neste exemplo, o tema da discussão é como adicionar uma meta tag do twitter card usando reat-helmet para renderizar dados dinamicamente no React. A issue possui um estado de closed, enquanto que a discussão no StackOverflow encontra-se aberta, sem respostas e votos.

Exemplo \#2. Na Figura 2 é exibida a relação entre a issue \#16241 ${ }^{4}$ encontrada no repositório ANGULAR/ANGULAR.JS e sua respectiva questão relacionada no StackOverflow. Neste exemplo, usuários do Github e do StackOverflow ${ }^{5}$ discutem sobre a funcionalidade de um método existente no projeto ANGULAR.JS, que retorna undefined quando atrelado a criação de link ou url. A issue atualmente encontra-se aberta no Github, assim como a discussão no StackOverflow. No entanto, a questão relatada no StackOverflow ainda não foi respondida e não recebeu nenhum voto da comunidade.

Exemplo \#3. A Figura 3 apresenta a relação entre a issue \#9830 ${ }^{6}$ encontrada no repositório METEOR/METEOR e sua respectiva questão relacionada no StackOverflow ${ }^{7}$. Neste exemplo, usuários do Github e do StackOverflow discutem sobre um problema ocorrido em um roteador chamado Iron Router que fez com ele parasse de funcionar.

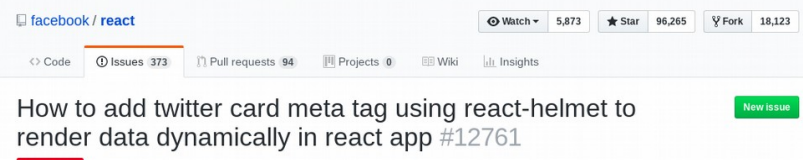
G. Closed championshuttler opened this issue 14 days ago 1 comment

Figura 1: Issue relacionada ao React

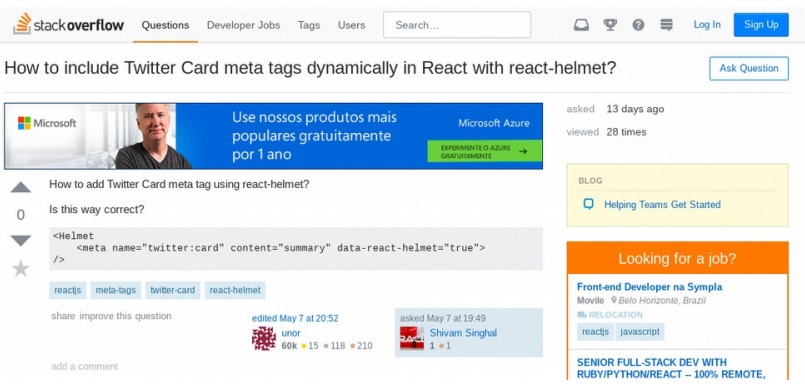

Figura 2: Questão relacionada do React

Iron Router é uma espécie de roteador que funciona em servidor e navegador e foi projetado especificamente para o METEOR. A issue atualmente encontra-se fechada, enquanto a discussão no StackOverflow encontra-se aberta

2https://goo.gl/yMCevG 3https://goo.gl/7TYV6m 4https://goo.gl/FYfKhL 5https://goo.gl/SQ1g1A 6https://goo.gl/vW9UWP 7https://goo.gl/LqMkYy 
com uma resposta e sem nenhum voto.

\section{B. Qual o impacto da discussão de issues no StackOverflow?}

Nessa questão de pesquisa investiga-se o impacto da discussão de issues no StackOverflow. Para avaliar tal impacto, foi utilizado o número de votos e respostas existente em cada uma das questões encontradas na questão de pesquisa QPA.

A tabela 3 sumariza os resultados encontrados para as doze questões identificadas no StackOverflow. Por meio desta tabela, percebe-se que issues não são muito discutidas no StackOverflow, uma vez que das doze perguntas encontradas apenas quatro questões foram respondidas gerando um feedback para o usuário criador. Além disso, percebe-se que as questões relacionadas às issues identificadas no StackOverflow não possuem uma alta popularidade, uma vez que tais questões possuem uma baixa quantidade de votos. Uma hipótese plausível que pode justificar esse baixo impacto é a diferença na comunidade que utiliza essas duas plataformas. Enquanto o Github engloba desenvolvedores que tem interesse em contribuir em um determinado projeto, o StackOverflow engloba usuários e desenvolvedores que desejam utilizar uma API em um projeto próprio.

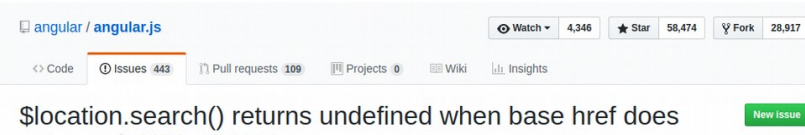
not match URL \#16241

(1) Open keturn opened this issue on 24 Sep 2017 . 4 comments

Figura 3: Issue relacionada ao AngularJS

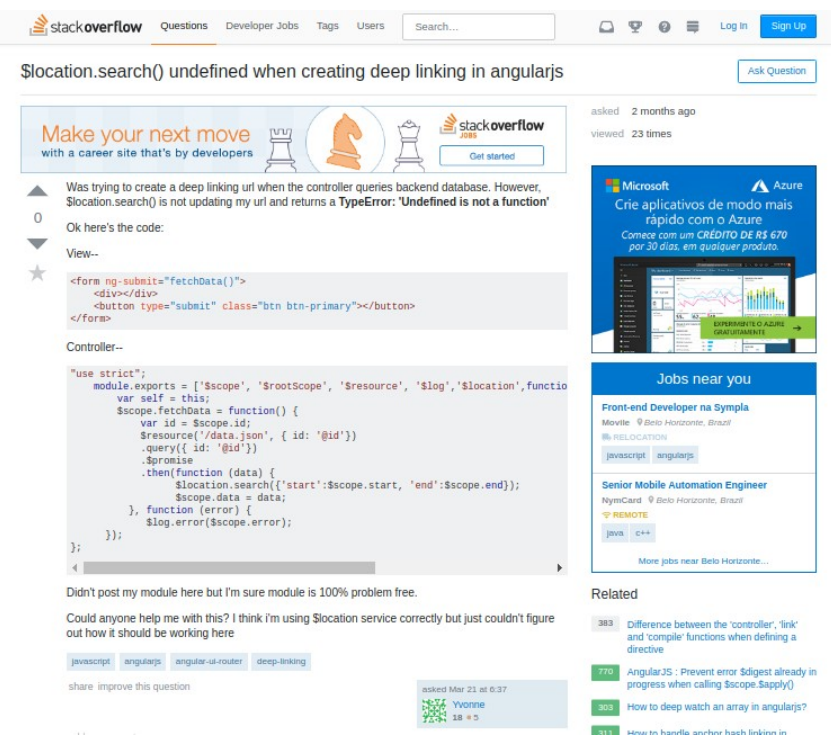

Figura 4: Questão relacionada do Angular JS

C. Existe uma relação entre as características dos sistemas e o buzz gerado no StackOverflow?

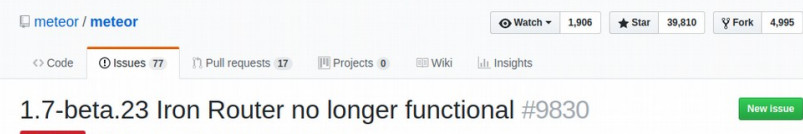

1.7-beta.23 Iron Router no longer functional \#9830

Figura 5: Issue relacionada ao Meteor

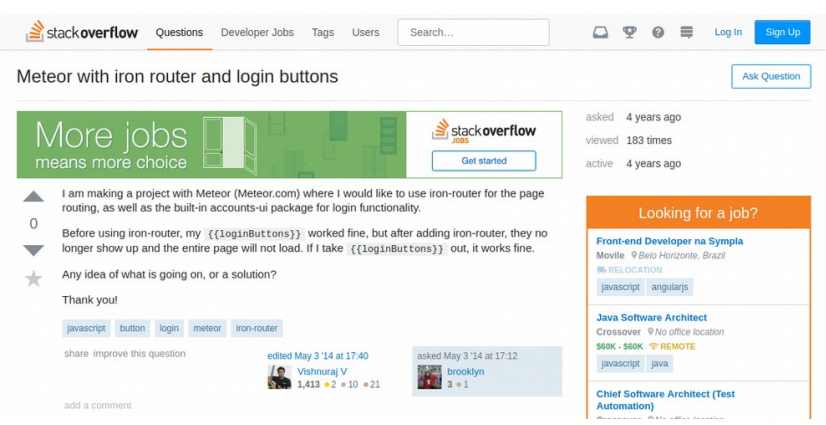

Figura 6: Issue e questão relacionada do Meteor

Esta questão de pesquisa tem como principal objetivo analisar se as características do sistemas analisados tem alguma influência sobre o buzz gerado no StackOverflow. Para respondê-la foi realizada uma análise sobre a popularidade dos sistemas, representado neste estudo pelo número de estrelas, e o número de issues e questões relacionadas identificadas no StackOverflow.

A tabela 4 apresenta os resultados identificados nessa questão de pesquisa, onde os quatro primeiros repositórios representam os mais populares e os quatro últimos representam os menos populares. Por meio desta tabela é possível observar que embora haja uma exceção, os repositórios mais populares foram os responsáveis pela geração de buzz no StackOverflow, ainda que tenha sido em uma pequena quantidade. O repositório Meteor/Meteor foi tratado com uma exceção neste estudo, uma vez que não foram identificadas questões no StackOverflow relacionadas às suas issues.

Embora não possa ser generalizado, os resultados desta questão de pesquisa indicam que os repositórios mais populares possuem uma tendência maior à geração de buzz no StackOverflow em comparação com os repositórios menos populares. Esse resultado pode ser explicado por dois motivos. Repositórios mais populares possuem uma maior quantidade de contribuidores em relação à repositórios menos populares. Além disso, repositórios mais populares possuem um maior relato de issues em relação à repositórios menos populares, indicando assim que eles estão em constante mudança.

D. Qual o perfil das pessoas que perguntam sobre issues no StackOverflow?

Nessa questão de pesquisa foi realizada uma comparação entre os autores das questões no StackOverflow e os reportantes das issues no Github, com a finalidade de verificar se possuem a mesma identidade. Após a verificação realizada, o resultado obtido foi que para todas as issues reportadas no Github, em caso algum seu reportante era o responsável por levantar sua questão relacionada no 
StackOverflow. Isso pode se dar devido a comunidade do StackOverflow para os repositórios analisados ser formada por usuários dos sistemas, em vez de desenvolvedores interessados nos projetos.

\begin{tabular}{|c|c|c|c|}
\hline$\#$ & Repositório & \#Respostas & \#Votos \\
\hline 1 & $\begin{array}{c}\text { Facebook / } \\
\text { React }\end{array}$ & 1 & 1 \\
\hline 2 & $\begin{array}{c}\text { Facebook / } \\
\text { React }\end{array}$ & 1 & 2 \\
\hline 3 & $\begin{array}{c}\text { Facebook / } \\
\text { React }\end{array}$ & 0 & 1 \\
\hline 4 & $\begin{array}{c}\text { Facebook / } \\
\text { React }\end{array}$ & 0 & 1 \\
\hline 5 & $\begin{array}{c}\text { Facebook / } \\
\text { React }\end{array}$ & 0 & 1 \\
\hline 6 & $\begin{array}{c}\text { Facebook / } \\
\text { React }\end{array}$ & 0 & 0 \\
\hline 7 & $\begin{array}{c}\text { Facebook / } \\
\text { React }\end{array}$ & 1 & 0 \\
\hline 8 & $\begin{array}{c}\text { Facebook / } \\
\text { React }\end{array}$ & 0 & 2 \\
\hline 9 & $\begin{array}{c}\text { Angular / } \\
\text { Angular.JS }\end{array}$ & 0 & 0 \\
\hline 10 & $\begin{array}{c}\text { Angular / } \\
\text { Angular.JS }\end{array}$ & 0 & 0 \\
\hline 11 & $\begin{array}{l}\text { Meteor / } \\
\text { Meteor }\end{array}$ & 0 & 1 \\
\hline 12 & $\begin{array}{l}\text { Meteor / } \\
\text { Meteor }\end{array}$ & 1 & 0 \\
\hline
\end{tabular}

Tabela 3: Quantidade de Respostas e Votos Referente as Questões

\begin{tabular}{|c|c|c|c|}
\hline Repositório & \#Estrelas & \#Issues & $\begin{array}{c}\text { \#Questões } \\
\text { relacionadas }\end{array}$ \\
\hline $\begin{array}{c}\text { Facebook/ } \\
\text { React }\end{array}$ & 95.893 & 224 & $8(2,7 \%)$ \\
\hline $\begin{array}{c}\text { Angular/ } \\
\text { Angular.JS }\end{array}$ & 58.430 & 90 & $2(2,2 \%)$ \\
\hline $\begin{array}{c}\text { JQuery/ } \\
\text { JQuery }\end{array}$ & 48.946 & 42 & $0(0 \%)$ \\
\hline $\begin{array}{c}\text { Meteor/ } \\
\text { Meteor }\end{array}$ & 39.789 & 112 & $2(1,8 \%)$ \\
\hline $\begin{array}{c}\text { Greglobinski/ } \\
\text { Gatsby- } \\
\text { Starter- } \\
\text { Personal- } \\
\text { Blog }\end{array}$ & 171 & 13 & $0(0 \%)$ \\
\hline \begin{tabular}{c} 
CacheContro \\
\hline
\end{tabular} & 128 & 0 & $0(0 \%)$ \\
\hline
\end{tabular}

\begin{tabular}{|c|c|c|c|}
\hline $\begin{array}{c}\text { l/Hippie- } \\
\text { Swagger }\end{array}$ & & 9 & $0(0 \%)$ \\
\hline $\begin{array}{c}\text { Marcrobledo/ } \\
\text { Savegame- } \\
\text { Editors }\end{array}$ & 102 & 1 & $0(0 \%)$ \\
\hline $\begin{array}{c}\text { HBM/MD- } \\
\text { Components }\end{array}$ & 101 & 9 & \\
\hline
\end{tabular}

Tabela 4: Quantidade de Questões Relacionadas a Issues

\section{TRABALHOS CORRELATOS}

Existem alguns métodos para vincular usuários de comunidades de software de código-aberto. Goeminne propôs um algoritmo para julgar se dois pares de usuários são a mesma pessoa [4]. Nosso trabalho usa o nome do usuário como chave principal para esta comparação e usamos informações textuais deixadas pelos desenvolvedores nas duas comunidades para melhorar a precisão.

No trabalho de Vasilescu et al, os autores concluem que a taxa de atividade do StackOverflow se correlaciona com a atividade de mudança de código no Github. Eles investigaram as interações entre as atividades do StackOverflow e o processo de desenvolvimento, refletido por alterações de código no Github. O estudo ainda mostra que os committers ativos do Github fazem menos perguntas e fornecem mais respostas do que outros. Além disso, destacam que os solicitantes do StackOverflow ativos distribuem seu trabalho de uma maneira menos uniforme do que os desenvolvedores que não fazem perguntas.

Silvestri et al, abordam em seu trabalho a capacidade de vincular perfis de usuários em várias redes sociais. Através de atributos do usuário, serviços específicos da plataforma e diferentes estratégias de correlação, eles contribuem com uma metodologia para vincular contas de usuário no StackOverflow e Github, além do Twitter. Tal metodologia pode ser usada em nosso trabalho para traçar com maiores informações os perfis dos usuários dessas redes.

Em contrapartida, o estudo de Xiong et al, descobre através de um proposta de metodologia para avaliar o comportamento de usuários no Github e no StackOverflow. Eles indicam que os committers ativos também são questionadores ativos, o que difere da amostragem do nosso estudo; para a maioria dos desenvolvedores, os tópicos de seus conteúdos no Github são semelhantes aos de suas perguntas e respostas no StackOverflow; as preocupações dos desenvolvedores no StackOverflow mudam ao longo do tempo de seus projetos participantes atuais no Github; as preocupações dos desenvolvedores no Github são mais relevantes para suas respostas do que perguntas e comentários no StackOverflow.

\section{AMEAÇAS À VALIDADE}

Validade Externa. Os resultados obtidos estão restritos a seis projetos JavaScript. Porém esse risco é atenuado pelo 
fato de os projetos analisados serem armazenados no Github, o repositório de código mais popular atualmente, e de fácil acesso.

Validade Interna. Os resultados apresentados podem ser afetados pela verificação manual da correspondência entre issues do Github e questões do StackOverflow realizada pelos autores. Para atenuar essa ameaça, cada autor conferiu as correspondências obtidas pelos demais.

Validade de Construção. As issues analisadas foram coletadas no intervalo de um mês. Logo, não se pode afirmar que os resultados obtidos refletem a completa reação entre issues do Github e buzz gerado no StackOverflow.

Validade de Conclusão. As conclusões do estudo estão sujeitas à confiabilidade das métricas escolhidas para responder as questões de pesquisa apresentadas. Para mitigar essa validade foram utilizadas métricas de repositório definidas no Github e métricas de questões definidas no StackOverflow. As métricas escolhidas são comumente utilizadas na literatura.

\section{CONCLUSÃO}

Neste trabalho, foi analisada a geração de buzz de issues do Github dentro do contexto de perguntas na comunidade do StackOverflow. Especificamente, o objetivo foi de (i) verificar se as issues reportadas no Github são também discutidas no StackOverflow; (ii) discutir o impacto dessas questões dentro do StackOverflow; (iii) avaliar se existe algum tipo de relação entre a popularidade dos sistemas analisados e o tamanho buzz gerado; e (iv) observar se os autores das questões encontradas são os mesmos das issues reportadas.

Como principais resultados, destaca-se que (i) poucas issues analisadas no Github são discutidas no StackOverflow; (ii) tais questões possuem um impacto pequeno dentro da comunidade do StackOverflow, com poucas respostas e baixa quantidade de votos; (iii) sistemas mais populares no Github tendem a possuir uma quantidade maior de perguntas relacionadas; e (iv) os usuários que reportam questionamentos no StackOverflow não são os mesmos que discutem issues nos repositórios analisados.

Por fim, destaca-se que os resultados deste estudo podem ser estendidos para outros contextos. Desta forma, como trabalhos futuros, planeja-se aumentar o tamanho do dataset analisado, a fim de aumentar a confiança das conclusões.
Além disso, planeja-se automatizar parte do processo de análise e coleta de dados, através a utilização de APIs próprias de mineração de informações do Github e do StackOverflow. Por fim, um estudo qualitativo envolvendo usuários que fazem questionamentos no StackOverflow apresenta-se como uma alternativa relevante para traçarmos o perfil das pessoas que participam da comunidade e discutem os mesmos tópicos reportados nas issues do Github.

\section{REFERÊNCIAS}

[1] Storey M. A. D. Treude C. Deursen A van. Cheng L. T. The impact of social media on software engineering practices and tools. Workshop on Future of software engineering research (FoSER). pp. 359-364. 2010.

[2] Capiluppi A. Serebrenik A. Singer L. Assessing technical candidates on the social web,” IEEE Software, vol. 30, no. 1, pp. 45-51, 2013.

[3] Dabbish L.A. Stuart H. C. Tsay J. Herbsleb J. D. Social coding in GitHub: transparency and collaboration in an open software repository. ACM Conference on Computer Supported Cooperative Work (CSCW). pp. 1277-1286. 2012.

[4] Montandon J. E. Politowski C. Silva L. L. Valente M. T. Petrillo F. Guéhéneuc Y-G. What Skills do IT Companies look for in New Developers? A Study with Stack Overflow Jobs. Information and Software Technology, vol. 1, pages 1-6, 2020.

[5] Abel F. Herder E. Houben G. J. Henze N. Krause D. Cross-system user modeling and personalization on the social web. User Modeling and User-Adapted Interaction. pp.169-209. 2013.

[6] Badashian A. S. Esteki A. Gholipour A. Hindle A. Stroulia E. Involvement, contribution and influence in github and stack overflow. International Conference on Computer Science and Software Engineering (CASCON). Pp:.19-33. ACM, 2014.

[7] Treude C. Barzilay O. Storey M. A. How do programmers ask and answer questions on the web?: Nier track. International Conference on Software Engineering (ICSE). pp. 804-807, IEEE, 2011.

[8] Robinson W. N. Deng T. Qi Z. Developer behavior and sentiment from data mining open source repositories. International Conference on System Sciences (HICSS). pp. 3729-3738, IEEE, 2016.

[9] Goeminne M. Mens T. A comparison of identity merge algorithms for software repositories,. Science of Computer Programming, vol. 78, no. 8, pp. 971-986. 2013.

[10] Vasilescu, B. Filkov, V. Serebrenik, A. StackOverflow and GitHub: Associations between Software Development and Crowdsourced Knowledge. International Conference on Social Computing. IEEE Computer Society, USA, pp. 188-195. 2013.

[11] Silvestri, G. Yang, J. Bozzon, A. Tagarelli et al. Linking Accounts across Social Networks: the Case of StackOverflow, Github and Twitter. KDWeb. 2015.

[12] Xiong, Y. Meng, Z. Shen, B. Yin, W. Mining Developer Behavior Across GitHub and StackOverflow. International Conference on Software Engineering \& Knowledge Engineerin (SEKE). pp. 578-583. 2017. 